CAHIERS

MONDES

ANCIENS

\section{Cahiers « Mondes anciens »}

Histoire et anthropologie des mondes anciens

$13 \mid 2020$

Qu'est-ce que faire école? Regards sur « l'école de

Paris »

\title{
Une école « géométrique » d'anthropologie historique dans le sillage de Durkheim
}

After Durkheim: A "Geometrical" School of Historical Anthropology

\section{Arnaud Macé}

\section{(2) OpenEdition}

\section{Journals}

Édition électronique

URL : http://journals.openedition.org/mondesanciens/2747

DOI : $10.4000 /$ mondesanciens. 2747

ISSN : 2107-0199

Éditeur

UMR 8210 Anthropologie et Histoire des Mondes Antiques

Référence électronique

Arnaud Macé, « Une école " géométrique » d'anthropologie historique dans le sillage de Durkheim », Cahiers « Mondes anciens » [En ligne], 13 | 2020, mis en ligne le 10 juin 2020, consulté le 12 juin 2020. URL : http://journals.openedition.org/mondesanciens/2747 ; DOI : https://doi.org/10.4000/ mondesanciens. 2747

Ce document a été généré automatiquement le 12 juin 2020.

\section{(†) $\odot$

Les Cahiers «Mondes Anciens » sont mis à disposition selon les termes de la licence Creative Commons Attribution - Pas d'Utilisation Commerciale - Pas de Modification 4.0 International. 


\title{
Une école " géométrique » d'anthropologie historique dans le sillage de Durkheim ${ }^{1}$
}

\author{
After Durkheim: A "Geometrical" School of Historical Anthropology
}

Arnaud Macé

1 La situation de l'anthropologie historique française reste, au début du XxI siècle, à tel point marquée par l'école animée, dans la deuxième partie du siècle précédent, par Jean-Pierre Vernant, qu'elle se trouve quelque peu à l'écart de l'un des débats qui animent plus généralement l'anthropologie, à savoir celui de son éventuel tournant «ontologique », conçu comme une manière de dépasser ou d'approfondir son moment structuraliste, par une extension du comparatisme aux cadres mêmes de l'enquête: désormais rien n'échappe à la nécessité d'une genèse empirique à partir des pratiques propres à chaque société, pas même les catégories comme celles de nature et de culture qui jusque-là pouvaient servir de cadre de référence au comparatisme lui-même ${ }^{2}$. Or, on peut soutenir que l'anthropologie historique de la Grèce ancienne n'a quant à elle pas connu le moment structuraliste à partir duquel la question du tournant ontologique est le plus souvent posée, par exemple au sein de l'anthropologie de la nature ${ }^{3}$. On a ainsi pu montrer, de manière convaincante, que la tentative d'application de l'analyse structurale des mythes à la Grèce ancienne, qui est précisément du fait de J.-P. Vernant et de son école, marque en réalité la distance qui demeure entre l'anthropologie historique et l'anthropologie structurale4. Nous voudrions suggérer dans la présente étude que la situation s'explique en grande partie par le fait que ladite «École de Paris » a maintenu en France l'anthropologie historique dans un rapport plus étroit à Durkheim et Mauss que les autres champs de la sociologie et de l'anthropologie. Ce décalage permet de reconnaitre la spécificité des questions soulevées par l'anthropologie de la Grèce ancienne et confère peut-être à celle-ci l'originalité d'un point de vue venu de plus loin, susceptible d'apporter sa pierre au débat ontologique qui anime aujourd'hui l'anthropologie, à condition de l'y introduire en tenant compte de cet écart. 


\section{Le moment géométrique de l'école de Paris}

2 La perception qu'une "école » existe suppose davantage que la contemporanéité et l'affinité des questionnements. Que l'on pense par exemple à l'affinité que l'on peut trouver, au début des années soixante, entre les hypothèses de Jean-Pierre Vernant, celles de James Goody et Ian Watt, d'un côté, et celles de Jürgen Habermas, de l'autre. Lorsque Jean-Pierre Vernant affirme en 1963 que l'écriture, en passant de la main des scribes des palais du Proche-Orient à celle des Grecs, connaît une modification dans sa «signification sociale »- passant du statut de privilège des scribes dans les palais des administrations royales du Proche-Orient au rôle de facteur d'accessibilité par l'affichage des lois et décrets ${ }^{5}$ - , il est le contemporain de Goody et Watt, qui, en 1963 aussi, insistent sur le fait que l'usage non syllabique de l'alphabet phénicien par les anciens Grecs aurait permis un processus de diffusion de l'écriture à travers une multiplicité inédites d'activités et de sphères sociales, stimulant par là aussi de nouvelles activités intellectuelles ${ }^{6}$. En affirmant en 1962 que «le caractère de pleine publicité donnée aux manifestations les plus importantes de la vie sociale » est un des traits fondamentaux de la cité grecque ${ }^{7}$, il est aussi le contemporain d'Habermas, qui, la même année, affirme que les évolutions politiques et historiques de grande ampleur résultent des modifications de l'öffentlichkeit, de la publicité au sens de l'accessibilité8. Tout cela ne fait pas une école. Ce qui en fait une, c'est la constance avec laquelle JeanPierre ${ }_{\text {vernant }}$ et ceux qui viendront travailler auprès de lui reprennent et développent ces thèses, en déployant un front collectif. On peut citer à cet égard la reprise par Marcel Detienne de la comparaison entre usage grec classique et usage hittite ou mycénien de l'écriture - il emploie, à propos de l'écriture, l'expression d'« opérateur de publicité ${ }^{\prime} »-$ ou celle de la question de l'affichage des lois sous le thème de l'écriture monumentale, pour affirmer le rôle du scribe grec comme «artisan de publicité » et non plus du secret ${ }^{10}$.

3 La dimension d'école tient aussi aux motifs particuliers autour desquels l'hypothèse de la publicité s'approfondit dans l'entourage de Jean-Pierre Vernant, et tout particulièrement le motif géométrique par lequel la nouvelle publicité de l'espace social trouve à se projeter dans l'espace de l'astronomie. Ce lien s'établit plus particulièrement autour du motif géométrique du centre et des points équidistants de la circonférence, qui constitue une structure commune à la représentation de l'Univers chez les présocratiques (en particulier chez Anaximandre) et à celle de la cité conçue dans les schèmes égalitaires de l'isonomie ${ }^{11}$. C'est avec Besançon que l'équipe parisienne de Jean-Pierre Vernant commence alors à faire école, lorsque, l'année suivante, paraît le livre de P. Lévêque et P.Vidal-Naquet, qui vient renforcer l'hypothèse d'un lien entre idées cosmiques et idées politiques ${ }^{12}$. Jean-Pierre Vernant insiste, dans le compte-rendu qu'il fait de cet ouvrage, sur la question de l'organisation spatiale, qui permet précisément de faire la médiation entre la cité et le $\operatorname{cosmos}^{13}$. Le "faire école» s'affirme avec la parution, dans le même numéro des Annales que le compte-rendu de J.-P. Vernant, d'un article de M. Detienne qui, lui aussi, approfondit la question des rapports entre politique et géométrie ${ }^{14}$, et s'ouvre sur une double référence à l'un et à l'autre ouvrage, celui de Lévêque et Vidal-Naquet et celui de Vernant. C'est en s'appuyant sur l'article de Marcel Detienne que Jean-Pierre Vernant pourra à son tour affirmer en 1968 que «les expressions $\dot{\varepsilon} \zeta \mu \varepsilon \dot{\varepsilon} \sigma 0 v, \dot{\varepsilon} v \mu \varepsilon \dot{\varepsilon} \sigma \omega$ sont 
exactement synonymes de $\dot{\varepsilon} \zeta$ koเvóv, $\dot{\varepsilon} v$ kolṿ̂̃. Le meson, le milieu, définit donc, par opposition à ce qui est privé, particulier, le domaine du commun, du public, le xunon ${ }^{15}$ ». Sans limiter l'ensemble des convergences qui permettent de parler d'une école de Paris, l'examen des publications des années soixante révèle ce qu'il faut reconnaître comme un paradigme commun, assez complexe, décrivant plusieurs couches de la réalité ancienne au moyen de configurations transversales : l'étude des motifs géométriques permet de lire l'histoire des pratiques politiques et l'histoire des idées cosmologiques comme les deux faces d'une même pièce.

\section{Une école cousine en milieu anglo-saxon? L'ascendance durkheimienne}

Ce modèle n'est pas sans postérité, mais n'a pas forcément survécu dans son intégrité : on trouvera l'étude de la correspondance entre formes politiques et formes cosmiques chez Antonio Capizzi, tandis qu'Anne-Gabrielle Wersinger a étudié pour eux-mêmes les motifs géométriques circulaires irriguant la pensée grecque de l'époque archaïque ${ }^{16}$. Le modèle lui-même n'est pas dénué d'antécédents. G. Vlastos, dans l'immédiat aprèsguerre, avait déjà cherché dans l'isonomie, l'égalité entre égaux, un motif commun à la pensée politique et cosmique des Anciens. Il le déclinait plus particulièrement à travers les représentations de la justice ${ }^{17}$. Il semble que ce style d'analyse, chez Vlastos, puisse être rapporté à un courant qui, dans les études anglo-saxonnes, remonte à Francis $\mathrm{M}$. Cornford et Jane E. Harrison. Ceux-ci, au début du siècle, s'employaient à déchiffrer la pensée cosmique et religieuse des Grecs à partir de leurs structures sociales. Or, c'était chez eux un modèle qu'ils empruntaient directement à Émile Durkheim et à Marcel Mauss: ce sont les sociologues français qui, aux yeux des deux antiquisants britanniques, fournissaient "la clef de la représentation religieuse » qui doit être recherchée " dans la structure sociale de la communauté qui l'élabore ${ }^{18}$, de telle sorte que l'on puisse se demander aussi, à propos des dieux grecs, "de quelle structure sociale ils sont la projection" (of what social structure are they the projection? ${ }^{19}$. On reconnaît ici la thèse centrale de l'essai de Mauss et Durkheim selon laquelle la transformation de la structure sociale en tant que telle, dans son rythme et dans son contenu, doit permettre de rendre compte des transformations des systèmes de classification $^{20}$. Ainsi Jane Harrison cherche le basculement du culte primitif de la déesse Thémis vers celui du panthéon olympien dans l'évolution qui mène la société indigène primitive, matriarcale, à une structure patriarcale imposée par les tribus venues du Nord: le rituel de l'initiation des Kourètes, dans la société matriarcale primitive, voit naître la figure de Zeus jeune homme, appelé à prendre place au sommet de l'organisation universelle dans les panthéons ultérieurs.

5 Nous voilà dès lors face à une hypothèse : s'il perdure encore après-guerre en milieu anglo-saxon un style de recherche sur la pensée ancienne qui hérite de la recherche des correspondances entre structures sociales et politiques et pensée cosmique, ne faut-il pas rapporter aussi l'école parisiano-bisontine de Vernant, Lévêque, Vidal-Naquet et Detienne à cette origine? Les deux formes d'interrogation seraient en réalité apparentées parce qu'elles remonteraient au même ancêtre. La filiation, côté français, semble facile à établir, puisque les deux «maîtres ${ }^{21}$ » de Jean-Pierre Vernant, Louis Gernet et Ignace Meyerson, constituent tous deux un chaînon possible dans l'héritage fût-il critique - de la sociologie de Durkheim et de Mauss. Jean-Pierre Vernant 
soulignait à quel point « l'influence durkheimienne » avait été constante chez Gernet, depuis la thèse de 1917 jusqu'aux ultimes études parue dans le Journal de psychologie normale et pathologique précisément dirigé par Meyerson, et se traduisait par :

[...] ce double et constant souci : partir des réalités collectives, à tous les niveaux, en cerner la forme dense, en bien mesurer le poids social, mais ne jamais les séparer des attitudes psychologiques, des mécanismes mentaux sans lesquels ni l'avènement, ni la marche, ni les changements des institutions ne sont intelligibles ${ }^{22}$.

6 Cette façon de reformuler l'héritage durkheimien de Gernet en faisant de la psychologie une partie intégrante du fait social est révélatrice, comme l'a bien vu Frédéric Fruteau de $\operatorname{Laclos}^{23}$, de la manière dont Vernant corrigeait Meyerson par Durkheim, à travers Gernet, en enracinant à nouveau la recherche des motifs mentaux dans la diversité des faits sociaux. Vernant, lestant la psychologie historique de Meyerson de nouveaux " ancrages matériels ${ }^{24}$ ", rétablit la question durkheimienne de l'articulation du social et du mental - il en rétablit même le sens en faisant des structures mentales à l'œuvre dans la pensée cosmologique la projection des structures à l'œuvre dans les faits sociaux. Ainsi la géométrie politique devient-elle l'ancrage matériel des géométries cosmiques.

\section{L'école de Paris relue au prisme de Durkheim}

Replacer plus explicitement le paradigme géométrique des années soixante dans l'héritage durkheimien permet à la fois de mieux mettre en relief certaines de ses spécificités, et peut-être d'en résoudre certaines apories. L'usage des motifs géométriques trouve là une certaine filiation. Gernet lui-même avait été attentif à certains motifs, comme ceux de la "mise au milieu», pour déchiffrer certaines pratiques du pré-droit des Grecs ${ }^{25}$. Durkheim et Mauss, dans l'essai sur les formes primitives de classification, ont eux-mêmes isolé le moment de l'évolution sociale où les structures claniques qui servent de matrice au classement de tous les choses et de tous les êtres se traduisent dans l'espace du camp ou du village, comme les sociétés amérindiennes en donnent l'exemple ${ }^{26}$, isolant ainsi le type de phase de l'évolution sociale qui est propice à ce que l'espace devienne un ancrage matériel pour les systèmes mentaux de classification.

Dans ce contexte, la façon dont l'école de Vernant développe ce style d'étude soulève plusieurs types de remarques ${ }^{27}$. Le modèle durkheimien perdure bien dans le fait que c'est le cœur de l'expérience sociale, dans son intersubjectivité rituelle, linguistique, juridique, politique ou simplement sensible (l'espace et le temps partagés), qui doit receler la clef de lecture des représentations du cosmos, précisément parce que ce sont ces moments d'intensification sociale qui permettent à la structure sociale de se détacher pour prendre les dimensions de l'univers. Ce modèle connaît néanmoins une forme de débordement chez Vernant, puisque les Grecs se voient reconnaître un certain degré de conscience de la différence entre le social et la nature à la faveur du développement d'un jeu analogique complexe entre ces deux niveaux ${ }^{28}$. Selon le modèle développé par Vernant, la rupture isonomique à l'œuvre dans la société, à travers l'onde de choc qui l'accompagne dans la modification du rapport au visible et à l'accessible, devient la matrice de projections analogiques vers l'univers : c'est le fait de la philosophie ionienne, qui représente dès lors une rupture avec la représentation hétérogène et hiérarchisée de l'espace mythique, et l'apparition d'une cosmogonie 
géométrique à l'espace homogène ${ }^{29}$. Si l'on prend l'exemple de l'analyse du rôle de la déesse Hestia ou de la position de la Terre dans la cosmologie d'Anaximandre, on peut dire que la projection d'un des systèmes mentaux vers l'autre, du social vers l'univers, est soutenu par trois arguments : l'homologie spatiale et fonctionnelle entre le foyer de la maison (Hestia domestique), le centre commun de la cité (Hestia commune) et la position de la Terre comme foyer de l'univers ${ }^{30}$; la correspondance du rythme des modifications dans le savoir cosmologique et des modifications sociales; le transfert qui signale l'origine politique du vocabulaire cosmique (la Terre en étant au centre n'est « dominée par rien d'autre (hupo mèdenos kratouménèn) », comme le pouvoir remis au centre est à tous ${ }^{31}$ ). Or, le modèle n'est pas exempt d'objections possibles sur les trois niveaux ${ }^{32}$.

9 Contre la correspondance des rythmes, on pourrait objecter la difficulté de prouver qu'au sein de cités soumises à des pouvoirs tyranniques comme le sont les cités d'Ionie, ce sont néanmoins des idéaux d'isonomie qui expliquent les nouveaux modèles cosmiques; ou, inversement, le fait que la représentation cosmique d'Hésiode manifeste déjà une recherche de symétrie tout à fait claire. Marcel Detienne a répondu à ces inquiétudes en ancrant l'isonomie et les configurations spatiales où elle s'illustrerait dans des pratiques aristocratiques antérieures à la démocratie, des pratiques qui outrepassent le champ politique strict - celle des jeux funéraires, du partage du butin ou de la délibération entre guerriers -, à moins précisément qu'elles n'en soient le cœur même, en tant que pratiques porteuses d'une rationalisation collective de la distribution des biens et des prérogatives ${ }^{33}$, réinscrivant l'évolution "démocratique» au sein d'une multiplicité de pratiques observables dans diverses cultures $^{34}$. Mais si l'on approfondit l'examen de ces pratiques distributives, on s'aperçoit aussi que l'idée du commun qu'elles nourrissent est plurivoque, et que cela rend ambiguës les figurations géométriques que l'on peut leur associer. Ainsi la circularité et le centre peuvent schématiser plusieurs formes du commun : la réserve commune, celle qui est préservée à l'écart des parts individuelles distribuées à chacun, afin de permettre des distributions futures par exemple; celles des choses communes distribuées, que l'on dit communes en tant que chacun en a eu une part égale ${ }^{35}$. Si la figure du cercle peut schématiser ces deux formes du commun, on ne peut plus se fier à la seule récurrence de tels motifs géométriques, qui doivent toujours être rapportés aux pratiques distributives sous-jacentes pour que leur sens s'éclaire.

\section{Revenir à Gernet : pour une philologie empirique}

Le troisième niveau, lexical, est lui aussi source de problèmes: Vernant cherchait à répondre à l'objection philologique qui lui avait été faite, selon laquelle le verbe $\kappa \rho \alpha \tau \varepsilon ́ \omega$ n'a pas toujours une signification politique - mais parfois simplement physique ${ }^{36}$. Or, il risque bien d'y avoir là une tentation de réduire les occurrences d'un terme à la signification qu'il porte dans l'un de ses domaines de référence. Louis Gernet a insisté pour que l'on reconnaisse le social dans ces phénomènes : on pourra expliquer qu'un mot change de sens en étant adopté par un groupe social plus large ou plus restreint que celui qui l'utilisait jusqu'alors, ou bien même que c'est le même groupe social dont la pensée s'altère lorsque se modifient les mots «que la société dans son ensemble fait siens $^{37} »$. La nature sociale des mots, nécessairement dispersés dans la multiplicité des actes de langages situés dans des sociétés, et dont les occurrences conservées portent 
l'empreinte, imposent à l'enquête anthropologique la nécessité de l'exercice philologique et lui imposent de pratiquer une certaine philologie. Le principe général en est celui de l'impossibilité de fixer le sens des occurrences d'un mot à partir d'une idée préconçue sur le sens et l'usage qui doivent prévaloir, ce qui suppose de se méfier $\mathrm{du}$ bons sens et de l'étymologie $\mathrm{e}^{38}$. Le bon sens pousse à dériver la signification en prenant modèle sur les processus de la psychologie individuelle, du particulier au général ou du général au particulier, ou encore sur l'association des idées ${ }^{39}$; l'étymologie promet de trouver à l'origine le sens général dont on veut tout dériver : celui-ci « dirige inconsciemment la recherche, et la racine une fois définie justifie le

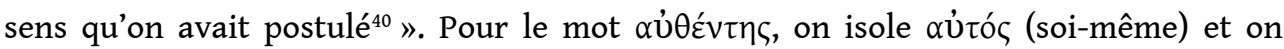
cherche une racine signifiant quelque chose comme «tuer ", en espérant dériver de là les différents sens que l'on croit trouver: meurtrier de soi, d'un parent, d'un autre, déduisant des "espèces différentes" dont nous ignorons pourtant si, dans «la psychologie effacée d'un groupe lointain », elles auraient "provoqué des sentiments du même ordre ou d'une commune mesure ${ }^{41} »$. Or l'enquête patiente, la lecture attentive de chacun des textes amène Gernet à balayer ces hypothèses, dissipées une à une, pour faire apparaître qu'au contraire l'idée que l'on s'en prend à quelqu'un de son clan, qu'il s'agit de «tuer l'un des nôtres ", est première, ce qui lui permet de renvoyer à l'analyse du rôle social des clans par Glotz ${ }^{42}$.

11 Le phénomène social de l'évolution des usages réclame une philologie empirique ${ }^{43}$, qui récuse trois niveaux d'intervention au moment de déterminer le sens d'un mot, à commencer par la logique et de la psychologie individuelle, ainsi que l'usage de l'étymologie motivé par celles-ci. Mais les facteurs externes sont aussi récusés : les événements historiques, l'histoire économique ou politique ne sont que des contextes permettant de déchiffrer le sens ponctuel d'un usage, mais en aucun cas des conditions permettant de généraliser un sens, car d'un point de vue empirique leur chronologie ne correspond pas au rythme des transformations sémantiques des notions morales, qui témoigne d'une «tranquillité silencieuse et comme souterraine qui ne s'affecte pas de l'histoire proprement dite ${ }^{44} »$. C'est l'ensemble de la refondation politique du modèle projectif qui était ainsi par avance mise en cause. Enfin, la façon dont Gernet ramène l'analyse linguistique au parcours de la somme des usages réels dont nous avons la trace exerce aussi un principe d'économie sur le terrain linguistique - cela referme par exemple la voie ouverte par Antoine Meillet de faire de la langue un fait social en s'appuyant sur la définition de structures syntaxiques en tant que telles, avec l'espoir de saisir par là des faits sociaux indépendamment du passage en revue des occurrences qui sont toujours soumises à la critique des philologues ${ }^{45}$. Avec Gernet, la syntaxe est un élément de plus pour comprendre la façon dont on fait usage des mots ${ }^{46}$. Il n'y a pas d'accès au social qui ne passe par des actes inscrits dans des sociétés : la langue ne peut être saisie qu'à partir des traces d'actes d'élocution et accomplis dans une certaine langue, dans une certaine période, dans une société donnée, traces auxquelles elle ne peut accéder sans la philologie que l'on vient de décrire. C'est sur ce terrain, en approfondissant la nature du lien qui unit a posteriori les différentes occurrences des mêmes termes, que l'alliance de la philologie et de l'anthropologie peut être, après Gernet, revivifiée.

$12 \mathrm{Au}$ fond cette inspiration empirique en philologie était peut-être déjà, chez Gernet, une façon d'être fidèle à Durkheim et à Mauss. Pour explorer le monde des Wakelbùra du Queensland-Nord-Central, il fallait chercher quelles sont les choses auxquelles ils 
attribuent les noms qui sont aussi ceux de leurs phratries, Mallera et Wùtarù : les porcs-épics, les kangourous ou les dindons sont dits "Mallera ", tandis que le serpent noir ou le miel des abeilles piquantes est « Wùtarù ». Ce premier temps de la recherche des classifications est déjà une philologie empirique, articulée à la recherche des affinités que les mots apparentés introduisent entre les choses ${ }^{47}$. Leur patient relevé s'impose peut-être comme la condition préalable à la saisie des classifications mentales à l'œuvre dans les pratiques sociales ${ }^{48}$. Tout se passe comme si le modèle projectif de la tradition durkheimienne, passé à travers l'anthropologie de la Grèce ancienne, autorisait celle-ci aujourd'hui à offrir aux nouvelles approches ontologiques de l'anthropologie une base empirique fondée sur une approche sociologique de la philologie. Elle y apporterait aussi une attention renouvelée aux ancrages matériels dans lesquelles des pratiques complexes parviennent parfois à se schématiser, ainsi les configurations spatiales et proto-géométriques dont la recherche a constitué l'un des apports originaux de l'école que nous venons de considérer.

\section{BIBLIOGRAPHIE}

Borecký B. (1965), Survivals of Some Tribal Ideas in Classical Greek: The Use and the Meaning of Lagchanō, Dateomai, and the Origin of Ison Echein, Ison Nemein, and Related Idioms, Prague.

Charbonnier P. (2015), La fin d'un grand partage. Nature et société de Durkheim à Descola, Paris.

charbonnier P., skafish P. et salmon G. éds. (2017), Comparative Metaphysics. Ontology after Anthropology, Lanham.

Cornford F. M. (1912), From Religion to Philosophy: A Study in the Origins of Western Speculation, Londres.

Descola P. (2005), Par-delà nature et culture, Paris.

- (2006), « Soyez réalistes, demandez l'impossible », L'Homme 177-178, 1, p. 429-434.

Detienne M. (1965), « En Grèce archaïque : géométrie, politique et société », Annales ESC 20-3, p. $425-441$.

- (1967), Les maîtres de vérité dans la Grèce archaïque, Paris.

- (1986), « L'écriture et ses nouveaux objets intellectuels en Grèce », Métis I-2, p. 309-324.

- (1988), Les savoirs de l'écriture en Grèce ancienne, Lille.

- (2003), Qui veut prendre la parole?, Paris.

Durkheim É. et Mauss M. (1903), « De quelques formes primitives de classification. Contribution à l'étude des représentations collectives ", L’Année sociologique 1901-1902, VI, p. 1-72.

Fruteau de Laclos F. (2012), «Vernant et Meyerson le mental, le social et le structural », Cahiers philosophiques 112, p. 9-25.

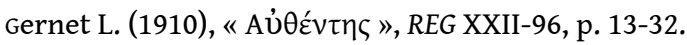


- (1917), Recherches sur le développement de la pensée juridique et morale en Grèce (Étude sémantique), Paris.

- (1947), « Jeux et droit (remarques sur le XXIIIe chant de l'Iliade) », CRAI 91-4, p. 572-574.

- (1968), Anthropologie de la Grèce antique, Paris.

Goody J. et Watt I. (1963), « The Consequences of Literacy », Comparative Studies in Society and History 5-3, p. 304-345.

Habermas J. (1962), Strukturwandel der Öffentlichkeit, Untersuchungen zu einer Kategorie der bürgerlichen Gesellschaft, Neuwied-Berlin ; trad. fr. L'espace public : archéologie de la publicité comme dimension constitutive de la société bourgeoise, 1978, Paris.

Harrison J. E. (1912), Themis: A Study of the Social Origins of Greek Religion, Cambridge.

Karsenti B. (2011), L'homme total. Sociologie, anthropologie et philosophie chez Marcel Mauss, Paris (1 1 ère éd. 1997).

Laks A. (2008), « Le génie du rapprochement et les limites de la similitude : à propos de l'Anaximandre de Vernant », Agenda de la pensée contemporaine 10, p. 113-127.

Lévêque P. et Vidal-Naquet P. (1964), Clisthène l'Athénien. Essai sur la représentation de l'espace et du temps dans la pensée politique grecque de la fin du $\mathrm{VI}^{e}$ siècle à la mort de Platon, Paris.

Macé A. éd. (2012), Choses privées et chose publique en Grèce ancienne. Genèse et structure d'un système de classification, Grenoble.

- éd. (2013), Le savoir public. La vocation politique du savoir en Grèce ancienne, Besançon.

- (2014), « Deux formes du commun en Grèce ancienne », Annales HSS 69-3, p. 659-688.

- (2017), « Nature among the Greeks: Empirical Philology and the Ontological Turn in Historical Anthropology », dans Charbonnier et al. 2017, p. 201-220.

salmon G. (2007), «Psychologie historique et analyse structurale chez J.-P. Vernant », Cahiers philosophiques 112-4, p. 42-65.

- (2013), Les structures de l'esprit : Lévi-Strauss et les mythes, Paris.

Sassi M. M. (2007), « Ordre cosmique et "isonomia” : en repensant Les origines de la pensée grecque de Jean-Pierre Vernant », Philosophie antique : problèmes, renaissances, usages 7, p. 191-215.

Vernant J.-P. (1962), Les origines de la pensée grecque, Paris.

- (1963), « Géométrie et astronomie sphérique dans la première cosmologie grecque », La pensée 109, p. 82-92; réimprimé dans Vernant 1965b, p. 201-215.

- (1965a), « Espace et organisation politique en Grèce ancienne », Annales ESC 20-3, p. 576-595.

- (1965b), Mythe et pensée chez les Grecs. Études de psychologie historique, Paris.

- (1968), «Structure géométrique et notions politiques dans la cosmologie d'Anaximandre », Eirene 7, p. 5-23.

Vlastos G. (1947), « Equality and Justice in Early Greek Cosmologies », CPh 42-3, p. 156-178.

Wersinger A. G. (2008), La sphère et l'intervalle. Le schème de l'harmonie dans la pensée des anciens Grecs d'Homère à Platon, Grenoble. 


\section{NOTES}

1. Le présent article reprend et prolonge certains résultats déjà parus dans une étude publiée en langue anglaise et inédite en français (мacé 2017). Plus particulièrement, certains paragraphes du présent article constituent l'original, enrichi sur plusieurs points, du texte traduit dans les pages 204 à 206 du chapitre en langue anglaise.

2. Sur la question du tournant ontologique de l'anthropologie, voir Charbonnier et al. 2017. Sur l'idée que l'approche ontologique, à savoir l'étude de la répartition des existants par chaque collectif, constitue une "métathéorie ", par rapport à laquelle la " cosmologie naturaliste ", c'est-à-dire "grosso modo, la répartition des êtres et des phénomènes entre l'universalité des lois de la nature et la contingence des productions humaines ", devient elle-même un agencement particulier, qui a certes accompagné la révolution scientifique mais «n'a revanche été démontrée de façon satisfaisante par aucune science officielle », voir Descola 2006, p. 433-434.

3. Ainsi par exemple Descola 2005.

4. Salmon 2007.

5. vernant 1963.

6. Goody et Watt 1963.

7. vernant 1962, p. 46, suivre tout le développement p. 44-48.

8. Habermas 1962.

9. Voir Detienne 1986, p. 314-315.

10. Detienne 1988, p.313-317. Sur l'ensemble des rapprochements de ce paragraphe, voir Macé 2013, «Introduction », p. 11-12.

11. Ce lien est affirmé dans le dernier chapitre de Vernant 1962, p.119-130, et il s'épanouit dans l'article (Vernant 1963) qui assimile nettement l'idée de «mettre au centre ", de rendre public et de mettre en commun (réimprimé dans Vernant 1965b, p. 201-215, voir particulièrement la p. 211 de cette édition).

12. Voir Lévêque et Vidal-Naquet 1964.

13. Voir Vernant 1965a, réimprimé dans Vernant 1965b, p. 238-260.

14. Detienne 1965.

15. Vernant 1968. L'ensemble des références rapprochées dans ce paragraphe l'ont été dans Macé 2014, note 15, p. 664.

16. Wersinger 2008.

17. Vlastos 1947.

18. Cornford 1912, p. viii.

19. Harrison 1912, p. 490.

20. Durkheim et Mauss 1903.

21. L'expression "notre maître " est employée par J.-P. Vernant à propos de Louis Gernet dans la première page de la préface qu'il rédige au volume Gernet 1968, p. 5. L'allégeance à Meyerson est magistralement proclamée dans la dédicace et le sous-titre de l'ouvrage de vernant 1965 b.

22. Gernet 1968, préface, p. 5 (p. 7-8 de la réédition Champs Histoire, Flammarion, 1995). 
23. Voir Fruteau de Laclos 2012, en particulier p. 22-23.

24. J'emprunte l'expression à Fruteau de Laclos 2012, p. 22.

25. Gernet 1947.

26. Durkheim et Mauss 1903, en particulier p. 47-53.

27. Les paragraphes qui suivent constituent l'original, inédit en français et enrichi sur plusieurs points, du développement paru en traduction anglaise aux pages 204 à 206 de Macé 2017.

28. Charbonnier 2015, p. 103-105.

29. Ibid., p. 100-103.

30. La conclusion de l'analyse d'Hestia est sans ambiguïté : «Ils ont donc projeté sur le monde de la nature l'image même de la société humaine dans la forme que la polis lui avaient conférée ", voir Vernant 1963 et réimpr. Vernant 1965b, p. 215. Elle est réaffirmée en 1968 à propos de la centralité de la Terre chez Anaximandre, voir Vernant 1968, p. 523 et réimpr. Vernant 1965b, p. 219.

31. La notion de pouvoir (kratos), transposée à la position de la Terre, «montre la persistance d'un vocabulaire et de concepts politiques dans la pensée cosmologique des Ioniens », Vernant 1962, p. 122.

32. Sassi 2007 ; Laks 2008.

33. Detienne 1967, chapitre V. Pour une étude plus générale des pratiques archaïques à l’origine des idées politiques des Grecs, voir Borecký 1965.

34. Detienne 2003.

35. Macé 2014.

36. L'article précédemment cité (Vernant 1968) tente de répondre à l'objection faite par Jan Janda (Eirene V, p. 205).

37. Gernet 1917, p. 423.

38. Lorsqu'il évoque les questions de méthode sémantique, dans le livre de 1917, Gernet renvoie à son article de 1910 (Gernet 1910). Ces écueils sont dénoncés au début de l'article de 1910 ; voyez aussi la reformulation du danger de la déduction dans la conclusion de l'ouvrage de 1917.

39. Gernet 1917, p. 424.

40. Gernet 1910, p. 14.

41. Ibid., p. 14.

42. La démonstration occupe l'ensemble de l'article, Glotz est invoqué p. 23.

43. L'expression elle-même n'est pas exactement sous la plume de Gernet, mais celui-ci défend une méthode fondée sur « l'histoire de mots ", pratiquée non pas « en se plaçant délibérément à certains points de vue », mais en la considérant « de façon empirique, sans savoir précisément où l'on veut aller, sans intention et, s'il faut le dire, sans parti pris », Gernet 1917, p. 4-5.

44. Ibid., p. 425.

45. Sur la liaison de ces deux objectifs voir Karsenti 2011, p. 158-169; sur la fortune de cette linguistique non philologique dans l'anthropologie, voir Salmon 2013, p. 57-86.

46. Gernet 1917, p. 425.

47. Durkheim et Mauss 1903, p. 10-12. 
48. Voir l'illustration d'une telle entreprise pour la représentation de l'individuel et du commun en Grèce ancienne dans Macé 2012, et pour une esquisse d'une recherche similaire sur les représentations grecques de la nature et du cosmos, voir Macé 2017

\section{RÉSUMÉS}

Le présent article entreprend de situer l'école qui s'est développée dans le sillage de Jean-Pierre Vernant au sein de l'héritage d'une autre école, celle d'Émile Durkheim. La contextualisation proposée s'ordonne à un motif particulier, celui de l'interprétation des idées cosmologiques à la lumière de la structure sociale des sociétés anciennes. On suit la diffusion d'une telle méthode dans les productions des années soixante de Jean-Pierre Vernant, Pierre Vidal-Naquet, Pierre Lévêque et Marcel Detienne, soit ce que l'on pourrait appeler une école de Paris-Besançon. On y repère la place prise par le motif géométrique, comme un schème assurant une lisibilité commune aux pratiques sociales et aux idées cosmologiques. Cela fait, on examine ce que ces développements doivent à Durkheim et à Mauss, à travers la médiation de Louis Gernet, tout en les comparant à la manière dont les philologues britanniques ont été inspirés par la sociologie française. Au total, cette proximité avec le modèle durkheimien donne à l'anthropologie historique de la Grèce ancienne une place à part dans les débats qui animent aujourd'hui l'anthropologie et les sciences sociales.

The present paper attempts to bring the school that developed after Jean-Pierre Vernant within the legacy of another, the school of Emile Durkheim. Such a contextualisation revolves around a particular aspect: the interpretation of cosmological ideas on the basis of the social structures of ancient societies. The paper follows the diffusion of such a method within the publications of the sixties by Jean-Pierre Vernant, Pierre Vidal-Naquet, Pierre Lévêque et Marcel Detienne - all in all what one could call a Paris-Besançon School. Particular attention is given to geometric patterns, used as a scheme providing a common intelligibility to social practices and cosmological ideas. Consequently, we try to assess what such developments owe to Durkheim and Mauss, through the mediation of Louis Gernet, also drawing a comparison with the way British philologists have received the inspiration of the French school of sociology. The proximity with the durkheimian model places historical anthropology of Ancient Greece in a particular place within the contemporary debate in anthropology and in social sciences.

\section{INDEX}

Mots-clés : Jean-Pierre Vernant, Pierre Vidal-Naquet, Pierre Lévêque, Marcel Detienne, Émile Durkheim, Marcel Mauss, cosmologie, géométrie, pratiques sociales

Keywords : Jean-Pierre Vernant, Pierre Vidal-Naquet, Pierre Lévêque, Marcel Detienne, Émile Durkheim, Marcel Mauss, cosmology, geometry, social practices 
AUTEUR

ARNAUD MACÉ

Université de Bourgogne Franche-Comté, Logiques de l'Agir EA 2274 